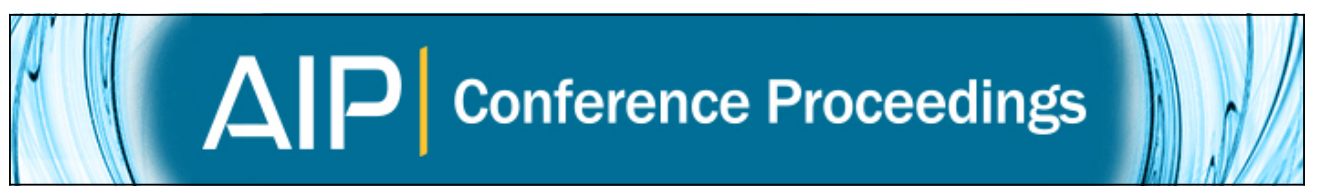

\title{
Finding Stellar Streams in Photometric Surveys
}

Carl J. Grillmair

Citation: AIP Conference Proceedings 1082, 226 (2008); doi: 10.1063/1.3059052

View online: http://dx.doi.org/10.1063/1.3059052

View Table of Contents:

http://scitation.aip.org/content/aip/proceeding/aipcp/1082?ver=pdfcov

Published by the AIP Publishing

\section{Articles you may be interested in}

The photometric properties and stellar populations in extremely red galaxies

AIP Conf. Proc. 1111, 199 (2009); 10.1063/1.3141543

Our Nearest 15 Million Neighbors: The Field Low—Mass Stellar Luminosity and Mass Functions

AIP Conf. Proc. 1094, 977 (2009); 10.1063/1.3099284

Searching for clusters and streams in large photometric surveys

AIP Conf. Proc. 1082, 233 (2008); 10.1063/1.3059054

SDSS analysis of Galactic stellar streams

AIP Conf. Proc. 1082, 221 (2008); 10.1063/1.3059051

Recent Results from the NOAO Fundamental Plane Survey

AIP Conf. Proc. 666, 315 (2003); 10.1063/1.1581810 


\title{
Finding Stellar Streams in Photometric Surveys
}

\author{
Carl J. Grillmair \\ Spitzer Science Center, 1200 E. California Blvd., Pasadena, CA 91125, U.S.A.
}

\begin{abstract}
The advent of high-quality, wide-area photometric databases has enabled significant advances in our knowledge of the structure, constituents, and accretion history of our Galaxy. Matched-filtering techniques allow us to map the spatial distributions of stellar populations with particular color-magnitude characteristics. This has already led to the detection of several long tidal streams in the Sloan Digital Sky Survey footprint. These streams are variously attributed to disrupted globular clusters and dwarf galaxies and offer us a new window into the structure, dark matter content, and formation history of the Galaxy. Similar techniques applied to future photometric surveys will enable us to expand the search for stellar debris streams over much of the Local Group.
\end{abstract}

Keywords: Galaxy: Structure - Galaxy: kinematics and dynamics — Galaxy: Halo: Galaxy — Tidal Streams

PACS: 98.35.Ln,98.35.Mp,98.35.Gi,98.35.Df

\section{INTRODUCTION}

As is often the case, experiments initially intended to pursue one set of scientific objectives can open up whole new areas of research. The arrival of large-area or all-sky photometric surveys such as the Sloan Digital Sky Survey (SDSS) and the Two-Micron All Sky Survey (2MASS), having been very successful in achieving their design goals, quickly enabled new (and sometimes unforeseen) investigations. The SDSS has proven to be a particular valuable resource for finding tidal streams and other Galactic substructures at a level of detail we cannot hope to match in any other galaxy. In addition to the large scale features attributed to past galaxy accretion events $[1,2,3,4,5,6,7,8]$, SDSS data has been used to detect the remarkably strong tidal tails of Palomar $5[9,10,11,12]$ and NGC $5466[13,14]$, as well as the presumed globular cluster streams GD-1 [15], Acheron, Cocytos, and Lethe [8]. Shown in Figure 1 is a composite stellar surface density map of some of the strongest streams detected in the SDSS footprint to date.

Though spectroscopic follow-up and detailed numerical calculations have yet to be carried out for most of these streams, they will ultimately become very important for constraining the three dimensional shape of the Galactic potential. Globular cluster streams will be particularly valuable since they are dynamically very cold [16] and therefore useful for constraining not only the global Galactic potential but also its lumpiness $[17,18]$.

\section{STAR COUNTS WITH A MATCHED FILTER}

Early efforts to divine the large scale distributions of stars from multi-color photometry relied on limiting the star counts to regions of color-magnitude space that are not 


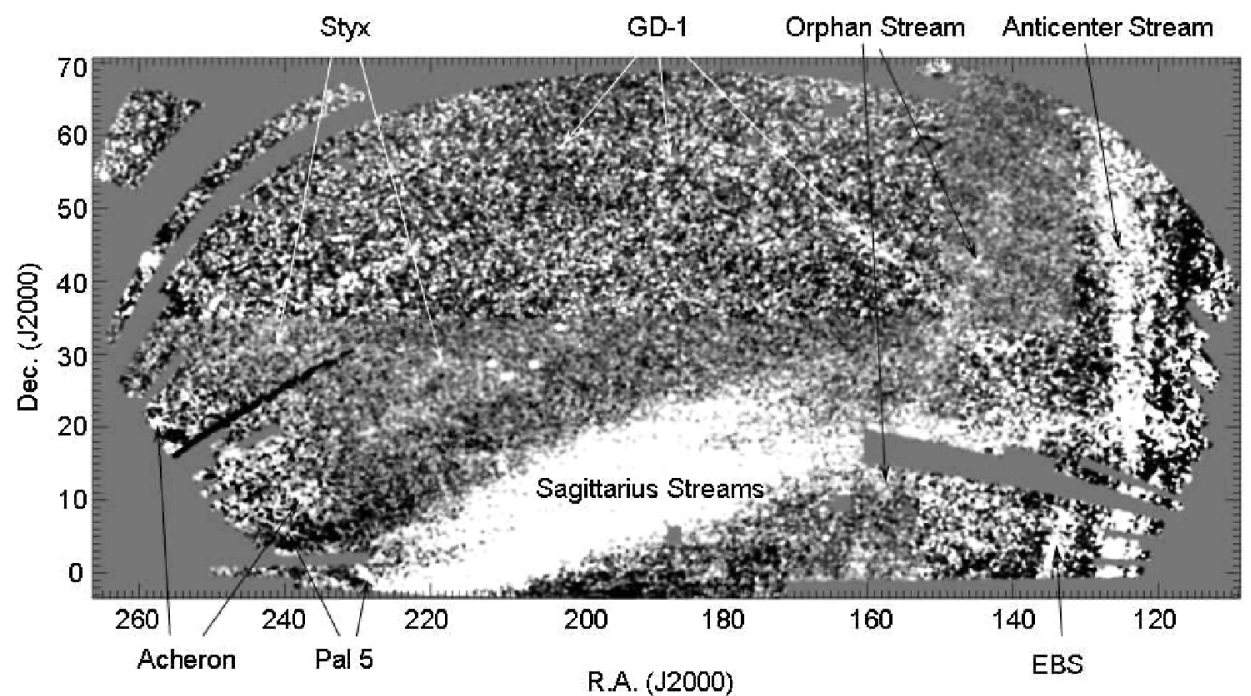

FIGURE 1. A smoothed, composite, filtered, stellar surface density map of the SDSS Data Release 5 footprint. The more pronounced streams are indicated. The survey area shown here has been been processed with a variety of different filters to enhance streams at different distances.

overwhelmed by foreground stars $[19,20,9,21]$. A substantial improvement on this method was pioneered by [10], who showed that the signal-to-noise ratio (SNR) could be significantly improved by employing an optimal matched filter. In essence, such a filter increases the weights applied to stars according to their likelihood of belonging to a particular stellar population, and deweights them according to the number of field stars occupying the same region of color-magnitude space. With such a weighting scheme, star counts can be extended well down the main sequence of the target population to where most of the stream stars live. This significantly enhances the visibility of very weak streams and helps to resolve dynamical substructures in more populous streams [6].

[10] applied this technique to a more detailed examination of the tidal tails of Pal 5, using the observed color-magnitude distribution (CMD) of stars in Pal 5 itself. However, if the goal is to find new streams of unknown pedigree and arbitrary distances, then a modified approach is required. Since any photometric survey will suffer varying degrees of incompleteness at faint magnitudes, it becomes necessary to separate the color-magnitude locus, the photometric uncertainties, the luminosity function, and the completeness function, and to recombine them in a manner appropriate to the search space under consideration. For example, $[4,6,8,15]$ used the CMDs of several globular clusters in the SDSS footprint to generate filters for a range of metallicities. An average completeness function was determined by comparing SDSS-observed luminosity functions in the outskirts of these globular clusters to much deeper luminosity functions from Hubble Space Telescope (HST) observations. The stellar luminosity function expected 


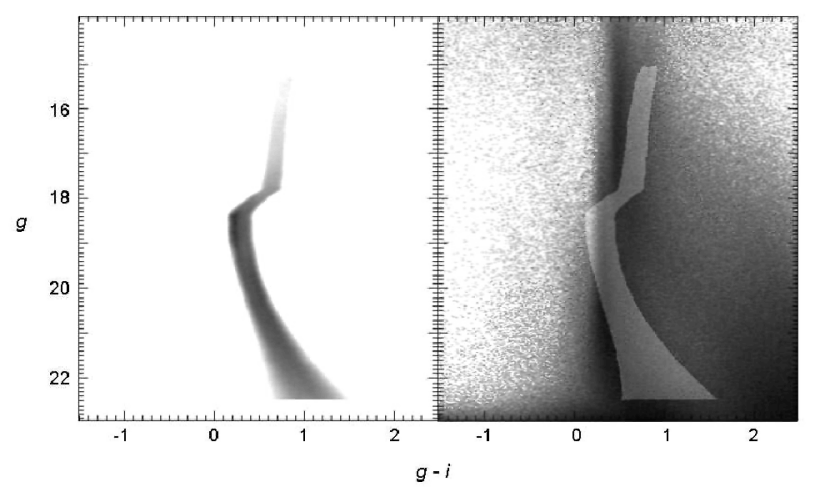

FIGURE 2. Panel (a): An example of a matched filter based on the SDSS color-magnitude distribution of stars in M 13, but with a luminosity function based on $\Omega$ Cen. The stretch is logarithmic. The peak response of the filter occurs on the blue side of the main sequence turnoff, where there are relatively few foreground stars. The subgiant and lower giant branch are deweighted due to the very large number off intervening foreground stars in this color range. The filter is Gaussian in cross section, with widths based on the mean SDSS photometric errors as a function of magnitude. Panel (b): The color-magnitude distribution of stars lying within 10 degrees of the eastern half of the Cocytos stream, with the filter in panel (a) overlaid.

in tidal streams is somewhat problematic as there are both dynamical arguments [22] and observations [23] to support the notion that tidal streams should contain a somewhat higher fraction of low mass stars. [8] used the luminosity function of $\omega$ Cen as measured with HST [24] and found that, due to the combined effects of survey incompleteness and greater field star contamination at faint magnitudes, the results were relatively insensitive to moderate increases in the fraction of low mass stars. Constructing a filter designed to search for a single-age population of a particular metallicity at a particular distance is then simply a matter of $(i)$ choosing the appropriate globular cluster template, (ii) vertically shifting both the CMD locus and luminosity function by the desired, clusterrelative distance modulus, and (iii) multiplying by the completeness function.

Constructing the optimal filter also requires a knowledge of the CMD of field stars. In searching the entirety of the Sloan survey area, [15] constructed a field CMD using stars over more than half the area of the survey. The advantage of this approach is that the CMD is very well and finely sampled over all colors and magnitudes likely to be of interest. The disadvantage is that the CMD may misrepresent the stellar population in the vicinity of a detected stream. Once a stream is detected, it is therefore usually beneficial (from an SNR standpoint) to modify the filter using the field star CMDs in the vicinity of various parts of the stream. An example of a typical filter based on the SDSS color-magnitude locus of M 13 at its nominal distance of $7.7 \mathrm{kpc}$ is shown in Figure 2. 


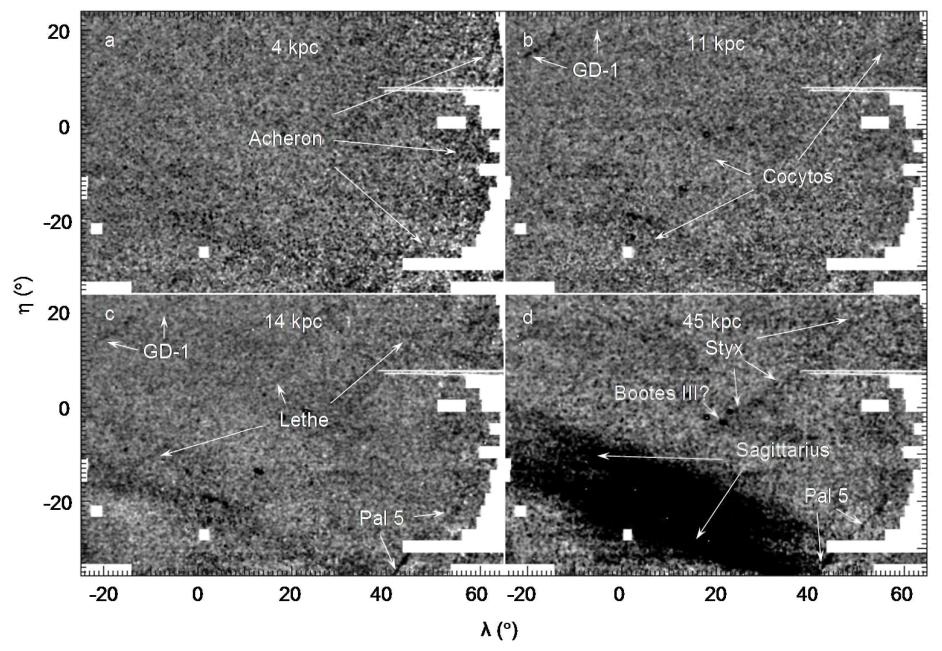

FIGURE 3. Weak streams recently detected [8] in the SDSS footprint. Three of these streams are probably remnants of globular clusters, with spatial widths of order $100 \mathrm{pc}$ or less.

\section{IDENTIFYING TIDAL STREAMS}

Owing perhaps to our hunter-gatherer origins, the human eye has a remarkable ability to discern structure in noisy fields. From experience, stellar streams are most easily distinguished by viewing a rapid sequence of filtered images in which the filter has been successively shifted to fainter and fainter magnitudes. The streams become apparent as linear features which often appear to move from one side of the survey area to the other as the sequence moves outward in distance. However, there comes a point when the identification of very faint streams by purely visual means becomes a highly subjective endeavor. Examples of some recently detected, very faint streams are shown in Figure 3.

To quantify the significance level of stream detections, [8] developed the T-statistic, which provides both a measure of the signal strength and of the continuity of the stream. In essence, a relatively short (e.g. 5 degree-long) window, aligned with and matched to the width of the putative stream, is moved laterally across a portion of the stream. A loworder polynomial is fitted to and subtracted from the run of signal strength with lateral offset to remove variations due to large scale Galactic structure. An identical operation is carried out for each segment along the stream's length. Finally, a median value for the background-subtracted signal strength is computed over all stream segments as a function of lateral offset. This last step is required to prevent compact structures (e.g. globular clusters) from strongly influencing the result. Figure 4 shows an example of the run of the T-statistic for the very weak Lethe stream in Figure 3. By comparing the signal strength of the stream with the RMS in the surrounding field, the SNR of the detection 


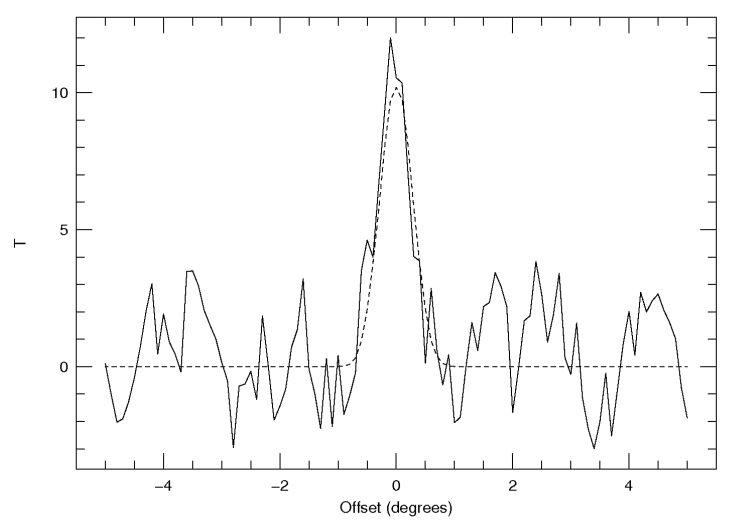

FIGURE 4. Median, background-subtracted, filtered signal strength in twelve, 5 degree-long by half degree-wide windows, as a function of lateral offset from the Lethe stream. Comparison with the surrounding field indicates that the Lethe stream (which has a mean surface density of $\sim 12$ stars per square degree with $g<22.5$ ) is detected at the $7 \sigma$ level. The dashed line shows the run of $T$ for an artificial stream at the same location, with a Gaussian cross section, and with a full-width-at-half-maximum of 0.4 degrees (or $95 \mathrm{pc}$ at a stream's distance of $13 \mathrm{kpc}$ ).

can be quantitatively estimated.

\section{STUDYING TIDAL STREAMS}

Once tidal streams have been detected and mapped, more focus can be brought to investigations of their properties, their numbers, and their implications for Galactic structure and formation. Even if the CMD of the stream is unmeasured, reasonable distance estimates can be made using just the main sequence portion of the search filter and choosing the distance modulus that maximizes the signal strength. If the stream has sufficiently high surface density, the CMD of the stream stars can be determined from the existing photometry. For example, Figure 5 shows a Hess diagram for stars in two tributaries of the Anticenter stream; the turn-off and main sequences are evidently very similar to that of the old, metal-poor globular cluster M 13.

For more tenuous streams, populating CMDs may require identification of individual stream stars through spectroscopic follow-up and kinematic association. Though costly in terms of telescope time, we expect a large number of such identifications to result from ongoing and near-term spectroscopic surveys such as SEGUE2, APOGEE, and LAMOST. Aside from unraveling the halo into its component streams and learning something about the progenitors, moderate resolution spectroscopy immediately enables sensitive studies of the three-dimensional shape of the Galactic potential. Since the stars in a tidal stream travel on very nearly the same orbit, sampling their velocities at widely separated positions enables a direct measure of $d \phi / d R$ and thus the mass distribution of the Galaxy. Figure 6 shows the orbit that best matches the position, velocity, and proper 


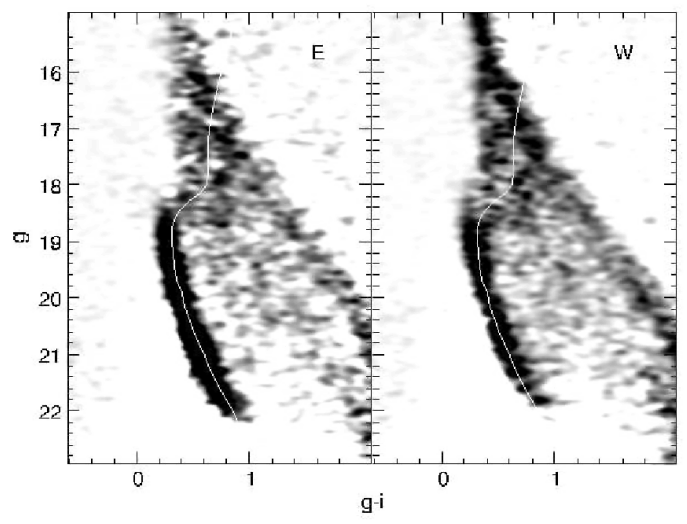

FIGURE 5. The color-magnitude distribution of stars in the central (C) and western (W) tributaries of the Anticenter Stream. The curves show the color-magnitude locus of stars in M 13, shifted vertically by +0.3 mags.

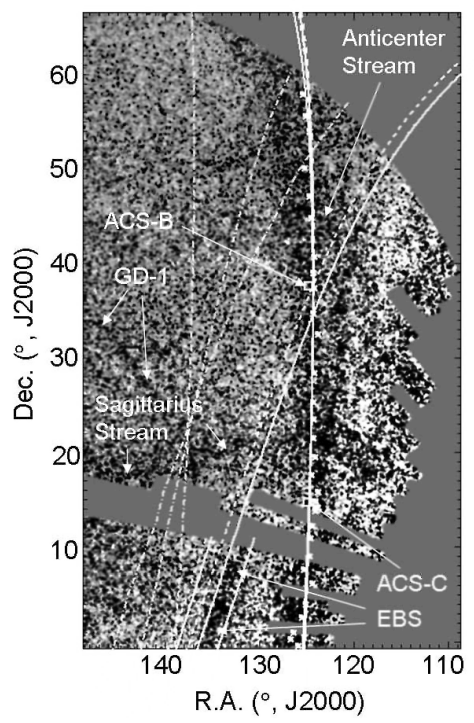

FIGURE 6. A projected orbit model for the Anticenter stream, based on radial velocity measurements made by [26] at the positions labeled ACS-B and ACS-C. A forward integration of the orbit overlays and is almost precisely aligned with the "Eastern Banded Structure" (EBS), suggesting that this stream-like feature is in fact the next wrap of the Anticenter stream. The dash-dot lines show projections of nearby wraps of the best-fit prograde orbits for the Monoceros stream[27]. 
motion of the Anticenter stream [8]. Using a relatively simple model of the Galactic potential[25], [8] show that the next wrap of the Anticenter stream coincides with the "Eastern Banded Structure", a stream-like feature noted by [6]. If this association is borne out by radial velocity measurements, the Anticenter stream will become only the third confirmed Galaxy-encircling stream known after Sagittarius[3] and Monoceros [21].

Still tighter constraints on the orbits of accreted remnants and on the shape of the Galactic potential will require significantly better proper motion measurements than are currently available. In this respect, GAIA, SIM-Lite, and LSST will contribute greatly to our understanding of the distribution of mass in the Galaxy, and of the hapless clusters and galaxies that have contributed to its build-up.

\section{REFERENCES}

1. R. Ibata, G. F. Lewis, M. Irwin, E. Totten, \& T. Quinn, Astrophys. J., 551, 294-311 (2001)

2. B. Yanny et al., Astrophys. J., 588, 824-841 (2003).

3. S. R. Majewski, M. F. Skrutskie, M. D. Weinberg, \& J. C. Ostheimer, Astrophys. J., 599, 1082-1115 (2003).

4. C. J. Grillmair, Astrophys. J., 645, L37-L40 (2006).

5. V. Belokurov et al., Astrophys. J., 642, L137-L140 (2006).

6. C. J. Grillmair, Astrophys. J., 651, L29-L32 (2006).

7. V. Belokurov et al., Astrophys. J., 658,337-344 (2007).

8. C. J. Grillmair, Astrophys. J., submitted, (2008).

9. M. Odenkirchen et al., Astrophys. J., 548, 165-169 (2001).

10. C. M. Rockosi, Astronom. J., 124, 349-363 (2002).

11. M. Odenkirchen et al., Astronom. J., 126, 2385-2407 (2003).

12. C. J. Grillmair, \& O. Dionatos, Astrophys. J., 641, L37-L39 (2006).

13. V. Belokurov, N. W. Evans, M. J. Irwin, P. C. Hewitt, M. I. Wilkinson, Astrophys. J., 637, L29-L32 (2006).

14. C. J. Grillmair, \& R. Johnson, Astrophys. J., 639, L17-L20 (2006).

15. C. J. Grillmair, \& O. Dionatos, Astrophys. J., 643, L17-L20 (2006).

16. F. Combes, S. Leon, \& G. Meylan, Astron. Astrophys, 352, 149-162 (1999)

17. C. Murali, \& J. Dubinski, Astronom. J., 118,911-919 (1999).

18. K. V. Johnston, D. N. Spergel, \& C. Hadyn, Astrophys. J., 570, 656-664 (2002)

19. C. J. Grillmair, K. C. Freeman, M. Irwin, P. J. Quinn, Astron. J., 109, $2555-2585$ (1995).

20. S. Leon, G. Meylan, \& F. Combes, Astron. Astrophys., 359, 907-931 (2000)

21. H. J. Newberg et al., Astrophys. J., 569, 245-274 (2002).

22. M. S. Fall \& Q. Zhang, Astrophys. J., 561, 751-765 (2001)

23. A. Koch, E. K. Grebel, M. Odenkirchen, D. Martinez-Delgado, \& J. A. R. Caldwell, Astronom. J., 128, 2274-2287 (2004)

24. G. de Marchi, Astronom. J., 117, 303-307 (1999)

25. C. Allen \& A. Santillan, Rev. Mex. Astron. Astrofis., 22, 255-263 (1991).

26. C. J. Grillmair, J. J. L. Carlin, \& S. R. Majewski, Astrophys. J., submitted, (2008).

27. J. Penarrubia, D. Martinez-Delgado, , H. W. Rix, M. A. Gomez-Flechoso, J. Munn, H. Newberg, E. F. Bell, B. Yanny, D. Zucker, \& E. K. Grebel, Astrophys. J., 626, 128-144 (2005). 